\title{
Kaposi Sarcoma in Mantled Guereza
}

\section{Anna Grewer, Martina Bleyer, Kerstin Mätz-Rensing, Alexander S. Hahn, Tim Rüggeberg, Gregor Babaryka, Andre Zimmermann, Stefan PöhImann, Artur Kaul}

We identified a novel Kaposi's sarcoma herpesvirus-related rhadinovirus (Colobine gammaherpesvirus 1) in a mantled guereza (Colobus guereza kikuyensis). The animal had multiple oral tumors characterized by proliferation of latent nuclear antigen 1-positive spindle cells and was not co-infected with immunosuppressive simian viruses, suggesting that it had Kaposi sarcoma caused by this novel rhadinovirus.

$\mathrm{K}$ aposi's sarcoma herpesvirus (KSHV), a member of the genus Rhadinovirus, is the causative agent of Kaposi sarcoma (1), an endothelial neoplasm of the dermis, oral cavity, and intestinal organs. The tumors are highly vascularized and characterized by proliferation of spindle cells that contain KSHV DNA and antigen $(2,3)$. Predisposing factors for Kaposi sarcoma include immunodeficiency, especially infection with HIV (4). Nevertheless, a major portion of Kaposi sarcoma cases in Africa occurs in HIVnegative persons (5).

Clinically, Kaposi sarcoma is divided into 4 forms: classical Kaposi sarcoma, African endemic Kaposi sarcoma, Kaposi sarcoma caused by iatrogenic immunosuppression, and HIV-associated Kaposi sarcoma (6,7). Lesions of classical Kaposi sarcoma initially occur on the lower extremities, progress slowly, and affect visceral organs at a late stage $(6,7)$. In contrast, the remaining Kaposi sarcoma forms affect lymph nodes, mucosa, and visceral organs at early stages, progress rapidly, and encompass symptoms in the hard palate and oral mucosa $(6,7)$.

Rhadinoviruses with high similarity to KSHV have been detected in Old World monkeys, including chimpanzees (8), macaques (9-11), and African green monkeys (12). The rhadinoviruses have split into 2 lineages, RV1 and RV2, and

Author affiliations: Zoo Krefeld $\mathrm{GmbH}$, Krefeld, Germany

(A. Grewer); German Primate Center-Leibniz Institute for Primate

Research, Göttingen, Germany (M. Bleyer, K. Mätz-Rensing,

A.S. Hahn, S. Pöhlmann, A. Kaul); Heinrich-Heine-University

Düsseldorf, Düsseldorf, Germany (T. Rüggeberg, G. Babaryka,

A. Zimmermann); Joint Practice for Oral and Maxillofacial Surgery,

Neuss, Germany (A. Zimmermann); University of Göttingen,

Göttingen (S. Pöhlmann)

DOI: https://doi.org/10.3201/eid2508.181804 many Old World monkeys harbor viruses of both lineages. In contrast, humans harbor only KSHV, which belongs to the RV1 lineage. Kaposi sarcoma-like disease has been observed in rhadinovirus-infected nonhuman primates (NHP), but only in the presence of immunodeficiency, induced, for instance, by co-infection with simian immunodeficiency virus $(13,14)$.

We report Kaposi sarcoma in a simian immunodeficiency virus- and simian retrovirus-negative mantled guereza (Colobus guereza kikuyensis) that was infected with a novel rhadinovirus that had high homology to KSHV. This new virus is called Colobine gammaherpesvirus 1 (CbGHV1).

\section{The Study}

A 13-year-old female mantled guereza who was born in a zoological garden in Germany showed development of swelling on the inner aspects of the lower lips; several circumscribed masses were found on the inner upper and lower labial mucosa. The masses were pink to light red and had a smooth and shiny surface, coarse consistence, and a diameter of 1-2 cm (Figure 1, panel A). After incision of 1 mass, the surface of the cut appeared to be cavernous and highly vascularized.

The mucosal masses were removed by surgery. Subsequently, bilateral cataract, progressive weight loss, and recurrence of the mucosal masses developed in the animal, and it had to be euthanized (Appendix, https://wwwnc. cdc.gov/EID/article/25/8/18-1804-App1.pdf). Necropsy showed several flattened and smooth tumorous lesions on the inner aspects of the upper and lower lips, as well as multiple small, partly ulcerated nodules at the gingival margin of the upper and lower jaw (Figure 1, panel B).

Histologically, masses and nodules consisted of a collagen-rich fibrous stroma with multifocal areas of increased cellularity represented by spindle cell proliferations with moderate lymphoplasmacellular infiltrates (Figure 1, panel C). The tonsils and mandibular and axillary lymph nodes showed similar foci of fibrovascular tissue. In the perinodal adipose tissue of 1 mandibular lymph node, we found distinct formation of caverns lined by endothelial cells, filled with erythrocytes, and surrounded by spindle cells (Figure 1, panel D).

Immunohistochemical examination showed distinct immunoreaction of most spindle cells with endothelial cell markers CD31 and von Willebrand factor. We found variable expression of Ki67 in $\leq 20 \%$ of spindle cells (Figure 1 , panel E), and $\approx 50 \%-60 \%$ of spindle cells reacted with 


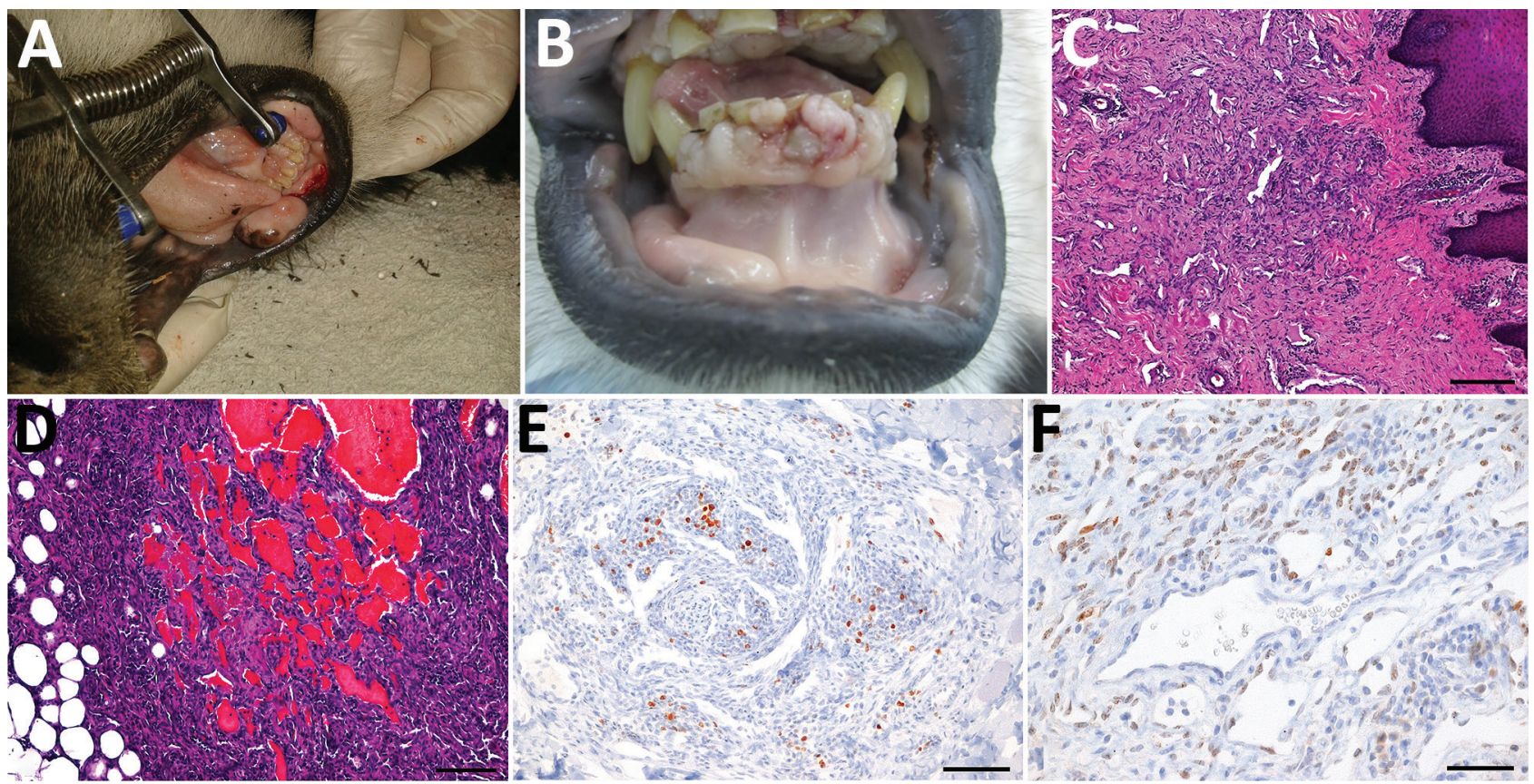

Figure 1. Disease manifestations in mantled guereza with Kaposi sarcoma. A) Oligofocal flattened masses on the inner aspects of the lower lip. B) Multinodular fissured masses at the gingival margin. C) Fibrovascular stroma in the subepithelial propria of the lower lip with spindle cell proliferations delineating narrow vascular clefts and containing lymphoplasmacytic inflammatory cell infiltrates, hematoxylin and eosin stained; scale bar indicates $200 \mu \mathrm{m}$. D) Spindle cell proliferation with cavern formation in the perinodal adipose tissue of the mandibular lymph node; hematoxylin and eosin stained; scale bar indicates $100 \mu \mathrm{m}$. E) Immunohistochemical staining showing variable Ki67 expression in $\leq 20 \%$ of spindle cells, streptavidin-biotin complex methoddiaminobenzidine tetrahydrochloride; scale bar indicates $100 \mu \mathrm{m}$. F) Immunohistochemical staining showing nuclear expression of latent nuclear antigen 1 in $\approx 50 \%-60 \%$ of spindle cells, streptavidin-biotin complex method-diaminobenzidine tetrahydrochloride; scale bar indicates $50 \mu \mathrm{m}$.

antibodies against KSHV latent nuclear antigen (Figure 1, panel F). These findings were compatible with Kaposi sarcoma. A pan herpesvirus PCR amplified DNA fragments in all tested samples (blood, swabs, pathologic tissues of upper and lower lips) that contained identical sequences of a novel herpesvirus (CbGHV1). This virus is most closely related to retroperitoneal fibromatosis herpesvirus from Macaca nemestrina (the pig-tailed macaque) (Figure 2, panel A) and was also identified in a sibling of the animal we studied (15).

We found high viral loads in the tumorous masses of the oral cavity, in swabs from cut surfaces of mucosal masses, and in tumorous lesions on the inner upper and lower lips, as well as in nodules at the gingival margin (Table 1). A lower viral load was detected in blood and was identical to that measured in the blood of a healthy offspring of the animal (Table 1). Viral load in all remaining organs was in the range of that measured for blood, potentially because of circulation of positive blood cells through these organs. Moreover, mucosal, anal, and fluid swab samples were clearly positive for viral genomes and the high viral load in the mucosa of the eye and in the lacrimal glands might be explained by Kaposi sarcoma in unusual locations (16). Finally, CbGHV1-negative samples were not available for calibration by PCR; their inclusion might have altered overall, but not relative, CbGHV1 genome copies measured.

Serologic analysis showed that the guereza had antibodies against lymphocryptovirus, cytomegalovirus, and simian foamy virus but, somewhat counter intuitively, not against rhesus rhadinovirus (RRV) (Table 2). In contrast, serum from the animal was reactive against KSHV antigen in an ELISA (Figure 2, panel B) and an immunofluorescence-based assay (data not shown). Finally, we did not detect antibodies reactive against KSHV in a CbGHV1-positive healthy offspring, potentially because CbGHV1 antibody levels were higher in diseased compared with healthy animals.

\section{Conclusions}

The animal we studied had several characteristic features of Kaposi sarcoma, including tumorous lesions in the buccal mucosa and proliferation of spindle cells, which harbored viral antigen. Although the disease symptoms did not fully match those of Kaposi sarcoma in humans $(3,4)$, in part because of absence of initial symptoms in the lower extremities, an animal model based on CbGHV1 might still provide major insights into Kaposi sarcoma/KSHV infection of humans. 
Table 1. Viral loads of CbGHV1 genomes in various organs of mantled guereza with Kaposi sarcoma*

\begin{tabular}{|c|c|}
\hline Category & $\begin{array}{c}\text { Copies of } \mathrm{CbGHV} 1 \\
\mathrm{DNA} / \mu \mathrm{g} \text { total } \mathrm{DNA} \pm \mathrm{SD}\end{array}$ \\
\hline \multicolumn{2}{|l|}{ Sampling during anesthesia } \\
\hline Blood & $4 \times 10^{4} \pm 8 \times 10^{3}$ \\
\hline Blood & $7 \times 10^{4} \pm 2 \times 10^{4} \dagger$ \\
\hline \multicolumn{2}{|l|}{ Mucosal masses } \\
\hline Swab specimen from cut surface & $6 \times 10^{7} \pm 3 \times 10^{7}$ \\
\hline Buccal & $9 \times 10^{6} \pm 1 \times 10^{6}$ \\
\hline Upper labial & $3 \times 10^{7} \pm 6 \times 10^{6}$ \\
\hline Lower labial & $2 \times 10^{7} \pm 3 \times 10^{6}$ \\
\hline \multicolumn{2}{|l|}{ Sampling during necropsy } \\
\hline \multicolumn{2}{|l|}{ Mucosal masses } \\
\hline Gingiva & $9 \times 10^{5} \pm 1 \times 10^{5}$ \\
\hline Upper labial & $6 \times 10^{6} \pm 1 \times 10^{6}$ \\
\hline Lower labial & $1 \times 10^{7} \pm 5 \times 10^{6}$ \\
\hline Oral swab specimen & $\begin{array}{l}5 \times 10^{3} \pm 2 \times 10^{3} \ddagger \\
5 \times 10^{4} \pm 2 \times 10^{4} \ddagger\end{array}$ \\
\hline Genital swab specimen & $6 \times 10^{4} \pm 1 \times 10^{4}$ \\
\hline Anal swab specimen & $3 \times 10^{5} \pm 5 \times 10^{4}$ \\
\hline Lacrimal fluid swab specimen & $3 \times 10^{6} \pm 6 \times 10^{5}$ \\
\hline Spleen & $5 \times 10^{5} \pm 9 \times 10^{4}$ \\
\hline Kidney & $6 \times 10^{3} \pm 3 \times 10^{3} \ddagger$ \\
\hline Kidney & $2 \times 10^{5} \pm 2 \times 10^{4} \ddagger$ \\
\hline Liver & $3 \times 10^{3} \pm 2 \times 10^{3}$ \\
\hline Lung & $1 \times 10^{4} \pm 6 \times 10^{3}$ \\
\hline Heart & $9 \times 10^{3} \pm 3 \times 10^{3}$ \\
\hline Brain & $1 \times 10^{4} \pm 5 \times 10^{3}$ \\
\hline $\begin{array}{l}\text { *Values are mean } \pm \text { SD for } 3 \text { independent } \\
\text { Colobine gammaherpesvirus } 1 \text {. } \\
\text { †Results for healthy offspring. } \\
\ddagger \text { Two samples were obtained during necr } \\
\end{array}$ & titative PCRs. CbGHV1, \\
\hline
\end{tabular}

CbGHV1 exhibited a higher similarity to KSHV and retroperitoneal fibromatosis herpesvirus from M. nemestrina, which are RV1 rhadinoviruses, when compared with RRV, a RV2 rhadinovirus. Consistent with these findings,

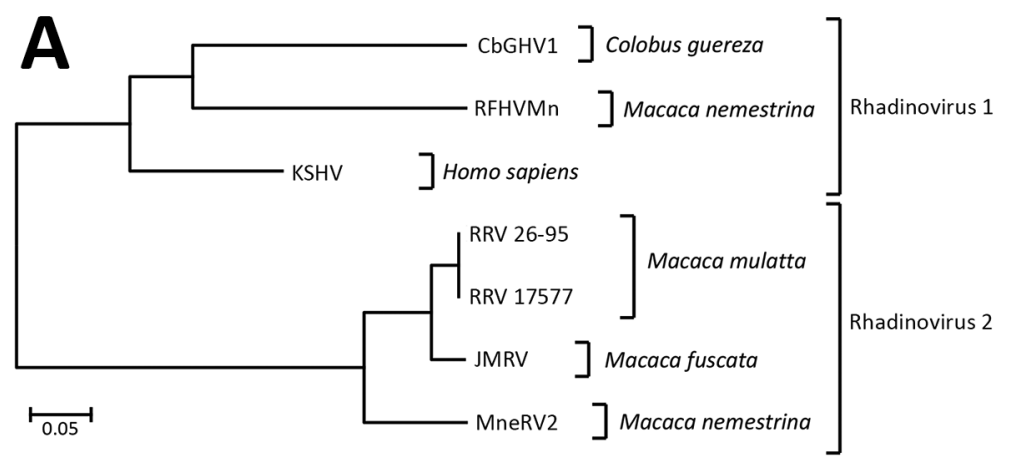

Table 2. Antibodies against selected viral antigens in mantled guereza with Kaposi sarcoma*

\begin{tabular}{lcc} 
& \multicolumn{2}{c}{ Test result } \\
\cline { 2 - 3 } Antigen source & $\begin{array}{c}\text { Monkey with } \\
\text { Kaposi sarcoma }\end{array}$ & $\begin{array}{c}\text { Healthy } \\
\text { offspring }\end{array}$ \\
\hline Herpes simplex viruses & - & - \\
Simian immunodeficiency virus & - & - \\
Simian retrovirus & - & - \\
Simian T-cell leukemia virus & - & - \\
Measles virus & - & - \\
Rhesus rhabdovirus & - & - \\
Lymphocryptovirus & + & + \\
Cytomegalovirus & + & + \\
Simian foamy virus & + & - \\
\hline${ }^{*}-$, negative; + , positive. & & \\
\hline
\end{tabular}

serum from the guereza cross-reacted with KSHV but not RRV. However, we cannot exclude that assay specificity was moderate and confirmation with independent tests is pending. Apart from the animal having Kaposi sarcoma, 4 genetically related animals were also PCR-positive for $\mathrm{Cb}$ GHV1, raising questions regarding the route of transmission. We detected high copy numbers of the viral genome in swab specimens of the oral cavity and the anogenital mucosa, suggesting that transmission might occur by close contact, including sex, the route of KSHV transmission between humans $(3,4)$.

CbGHV1 was most likely involved in tumorigenesis because high numbers of the viral genome were found within tumorous tissues. Moreover, viral antigen was detected in spindle cells. However, it was unclear that infection by CbGHV1 was sufficient to induce Kaposi sarcoma. A link between immunosuppression and Kaposi sarcoma has been established for human patients and cannot be excluded for

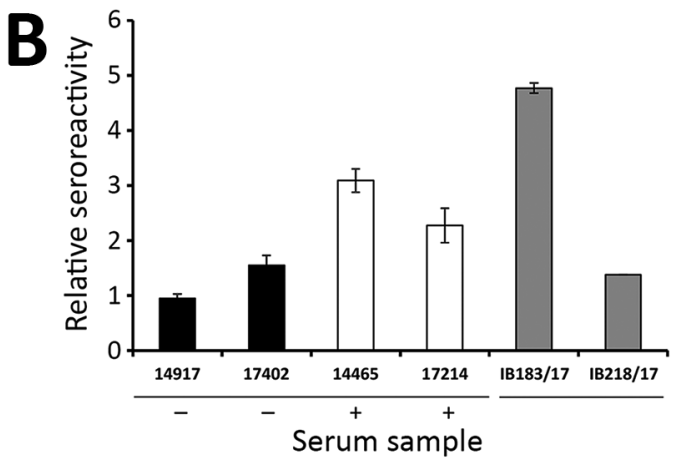

Figure 2. Analysis of CbGHV1 and seroreactivity in mantled guereza with Kaposi sarcoma. A) Phylogenetic analysis of partial sequences of the polymerase gene. Analysis was performed by using the neighbor-joining method. The distance between CbGHV1 and selected viruses was analyzed by using the maximum composite-likelihood method and MEGA6 (https://www.megasoftware.net). The PCR sequence of CbGHV1 was compared with KSHV (GenBank accession no. NC_009333.1); RFHVMn (KF703446.1); RRV 26-95 (AF210726.1); RRV 17577 (NC_003401.1); JMRV (AY528864.1); and MneRV2 (KP265674.2). Scale bar indicates nucleotide substitutions per site. B) Antibodies from mantled guereza with Kaposi sarcoma showing cross-reactivity against KSHV. Reactivities of KSHV antibody-positive human serum samples (14465 and 17214), KSHV antibody-negative human serum samples (14917 and 17402), and serum sample from the Kaposi sarcomaaffected mantled guereza (IB183/17) and its healthy offspring (IB218/17) were analyzed by ELISA. Relative reactivities of serum samples with KSHV-positive and KSHV-negative cell lysates are shown. The sum of relative errors is used as an error estimate for the ratio and is indicated by error bars (mean \pm half error). Reactivity of human serum samples against KSHV is indicated. CbGHV1, Colobine gammaherpesvirus 1; JMRV, Japanese macaque rhadinovirus; KSHV, Kaposi's sarcoma herpesvirus; MneRV2, Macaca nemestrina rhadinovirus 2; RFHVMn, retroperitoneal fibromatosis-associated herpesvirus $M$. nemestrina; RRV, rhesus rhadinovirus; -, negative; +, positive. 
CbGHV1/NHP $(3,4)$. A younger male sibling of the guereza analyzed in this study was PCR positive for CbGHV1 and showed development of primary effusion lymphoma, another disease caused by KSHV, without evidence for immunosuppression (15). Thus, a genetic component might contribute to disease development. Finally, 1 offspring of the animal infected with Kaposi sarcoma and 2 genetically related animals were also CbGHV1-positive but healthy; it remains to be examined whether they will show development of disease in the future.

We report a case of spontaneous Kaposi sarcoma in an NHP. Our findings might aid the development of an NHP model for KSHV/Kaposi sarcoma in humans. For development of this model, it is critical to isolate CbGHV1; those efforts are under way.

\section{Acknowledgments}

We thank Klaus Korn for providing serum samples from KSHV+ and KSHV- patients and Bastian-Felix Danitz, Sarah Schlagowski, Nadine Schminke, and Larissa Hummel for providing excellent technical assistance.

\section{About the Author}

Dr. Grewer is a veterinarian at the Zoo Krefeld, Krefeld, Germany. Her primary research interest is infectious diseases in zoo animals.

\section{References}

1. Moore PS, Chang Y. Detection of herpesvirus-like DNA sequences in Kaposi's sarcoma in patients with and those without HIV infection. N Engl J Med. 1995;332:1181-5. https://doi.org/10.1056/ NEJM199505043321801

2. Boshoff C, Schulz TF, Kennedy MM, Graham AK, Fisher C, Thomas A, et al. Kaposi's sarcoma-associated herpesvirus infects endothelial and spindle cells. Nat Med. 1995;1:1274-8. https://doi.org/10.1038/nm1295-1274

3. Mariggiò G, Koch S, Schulz TF. Kaposi sarcoma herpesvirus pathogenesis. Philos Trans R Soc Lond B Biol Sci. 2017; 372:20160275. https://doi.org/10.1098/rstb.2016.0275

4. Yarchoan R, Uldrick TS. HIV-associated cancers and related diseases. N Engl J Med. 2018;378:1029-41. https://doi.org/ 10.1056/NEJMra1615896

5. Host KM, Horner MJ, van der Gronde T, Moses A, Phiri S, Dittmer DP, et al. Kaposi's sarcoma in Malawi: a continued problem for HIV-positive and HIV-negative individuals.
AIDS. 2017;31:318-9. https://doi.org/10.1097/QAD. 0000000000001341

6. Antman K, Chang Y. Kaposi's sarcoma. N Engl J Med. 2000; 342:1027-38. https://doi.org/10.1056/NEJM200004063421407

7. Friedman-Kien AE, Saltzman BR. Clinical manifestations of classical, endemic African, and epidemic AIDS-associated Kaposi's sarcoma. J Am Acad Dermatol. 1990;22:1237-50. https://doi.org/ 10.1016/0190-9622(90)70169-I

8. Greensill J, Sheldon JA, Murthy KK, Bessonette JS, Beer BE, Schulz TF. A chimpanzee rhadinovirus sequence related to Kaposi's sarcoma-associated herpesvirus/human herpesvirus 8: increased detection after HIV-1 infection in the absence of disease. AIDS. 2000;14:F129-35. https://doi.org/10.1097/ 00002030-200012010-00001

9. Desrosiers RC, Sasseville VG, Czajak SC, Zhang X, Mansfield KG, Kaur A, et al. A herpesvirus of rhesus monkeys related to the human Kaposi's sarcoma-associated herpesvirus. J Virol. 1997;71:9764-9.

10. Rose TM, Strand KB, Schultz ER, Schaefer G, Rankin GW Jr, Thouless ME, et al. Identification of two homologs of the Kaposi's sarcoma-associated herpesvirus (human herpesvirus 8) in retroperitoneal fibromatosis of different macaque species. J Virol. 1997;71:4138-44.

11. Strand K, Harper E, Thormahlen S, Thouless ME, Tsai C, Rose $\mathrm{T}$, et al. Two distinct lineages of macaque gamma herpesviruses related to the Kaposi's sarcoma associated herpesvirus. J Clin Virol. 2000;16:253-69. https://doi.org/10.1016/ S1386-6532(99)00080-3

12. Greensill J, Sheldon JA, Renwick NM, Beer BE, Norley S, Goudsmit J, et al. Two distinct gamma-2 herpesviruses in African green monkeys: a second gamma-2 herpesvirus lineage among old world primates? J Virol. 2000;74:1572-7. https://doi.org/10.1128/ JVI.74.3.1572-1577.2000

13. Orzechowska BU, Powers MF, Sprague J, Li H, Yen B, Searles RP, et al. Rhesus macaque rhadinovirus-associated non-Hodgkin lymphoma: animal model for KSHV-associated malignancies. Blood. 2008;112:4227-34. https://doi.org/10.1182/ blood-2008-04-151498

14. Wong SW, Bergquam EP, Swanson RM, Lee FW, Shiigi SM, Avery NA, et al. Induction of B cell hyperplasia in simian immunodeficiency virus-infected rhesus macaques with the simian homologue of Kaposi's sarcoma-associated herpesvirus. J Exp Med. 1999;190:827-40. https://doi.org/10.1084/jem.190.6.827

15. Dhingra A, Ganzenmueller T, Hage E, Suárez NM, Mätz-Rensing K, Widmer D, et al. Novel virus related to Kaposi sarcoma-associated herpesvirus from Colobus monkey. Emerg Infect Dis. 2019;25:1548-51.

16. Pantanowitz L, Dezube BJ. Kaposi sarcoma in unusual locations. BMC Cancer. 2008;8:190. https://doi.org/10.1186/1471-2407-8-190

Address for correspondence: Anna Grewer, Zoo Krefeld GmbH,

Uerdinger Strasse 377, Krefeld 47800, Germany; email:

anna.grewer@zookrefeld.de 